\title{
Factors Controlling the Barriers to Degenerate Hydrogen Atom Transfers
}

\author{
Christine Isborn, David A. Hrovat, Weston Thatcher Borden*, James M. Mayer*, \\ and Barry K. Carpenter* \\ Contribution from the Department of Chemistry, University of Washington, Seattle, WA \\ 98195-1700 and the Department of Chemistry and Chemical Biology, Baker Laboratory, \\ Cornell University, Ithaca, NY 14853-1301.
}

\section{Supporting Information}

1. Details of Electronic Structure Calculations.

All calculations were carried out with the Gaussian 03 program package. ${ }^{1}$ Density functional calculations employed the MPW1K functional of Truhlar et $a l .^{2}$ and used the $6-31+G(d, p)$ basis set. ${ }^{3}$ Complete-basis-set calculations used the CBS-QB3 model. ${ }^{4}$ UCCSD(T) geometry optimizations were carried out with the cc-pVTZ basis set $^{5}$ and used numerical gradient evaluations. Cartesian coordinates (in $\AA$ ), energies and enthalpies (both in hartree) of each stationary point are listed below.

1.1.1 MPW1K/6-31+G(d,p) results:

$$
\underline{1.1 \mathrm{CH}_{4}}
$$

Cartesian Coordinates

$\begin{array}{rrrr}\mathrm{C} & 0.000000 & 0.000000 & 0.000000 \\ \mathrm{H} & 0.626625 & 0.626625 & 0.626625 \\ \mathrm{H} & -0.626625 & -0.626625 & 0.626625 \\ \mathrm{H} & -0.626625 & 0.626625 & -0.626625 \\ \mathrm{H} & 0.626625 & -0.626625 & -0.626625\end{array}$

Energy: -40.512919

Enthalpy: -40.463109

\subsubsection{CBS-QB3 results:}

$\begin{array}{lrrr} & & \text { Cartesian } & \text { Coordinates } \\ \mathrm{C} & 0.000000 & 0.000000 & 0.000000 \\ \mathrm{H} & 0.629726 & 0.629726 & 0.629726 \\ \mathrm{H} & -0.629726 & -0.629726 & 0.629726 \\ \mathrm{H} & -0.629726 & 0.629726 & -0.629726 \\ \mathrm{H} & 0.629726 & -0.629726 & -0.629726\end{array}$

Energy: -40.454142
Enthalpy: -40.406184

1.1.3 CCSD(T)/cc-pVTZ results:

$\begin{array}{lrrr} & & \text { Cartesian Coordinates } \\ \text { C } & 0.000000 & 0.000000 & 0.000000\end{array}$




$\begin{array}{rrrr}\mathrm{H} & 0.628740 & 0.628740 & 0.628740 \\ \mathrm{H} & -0.628740 & -0.628740 & 0.628740 \\ \mathrm{H} & -0.628740 & 0.628740 & -0.628740 \\ \mathrm{H} & 0.628740 & -0.628740 & -0.628740\end{array}$

Energy: -40.4380994

1.2.1 MPW1K/6-31+G(d,p) results:

\section{$\underline{1.2 \mathrm{CH}_{3}}-$}

Cartesian Coordinates

$\begin{array}{rrrr}\mathrm{C} & 0.000000 & 0.000000 & 0.000000 \\ \mathrm{H} & 0.000000 & 1.075848 & 0.000000 \\ \mathrm{H} & 0.931712 & -0.537924 & 0.000000 \\ \mathrm{H} & -0.931712 & -0.537924 & 0.000000\end{array}$

Energy: -39.835679

Enthalpy: -39.801117

\subsubsection{CBS-QB3 results:}

$\begin{array}{lrrr} & & \text { Cartesian } & \text { Coordinates } \\ \mathrm{C} & 0.000000 & 0.000000 & 0.000000 \\ \mathrm{H} & 0.000000 & 1.080624 & 0.000000 \\ \mathrm{H} & 0.935848 & -0.540312 & 0.000000 \\ \mathrm{H} & -0.935848 & -0.540312 & 0.000000\end{array}$

Energy: -39.774073

Enthalpy: -39.740784

1.2.3 UCCSD(T)/cc-pVTZ results:

$\begin{array}{lrrr} & & \text { Cartesian } & \text { Coordinates } \\ \mathrm{C} & 0.000000 & 0.000000 & 0.000000 \\ \mathrm{H} & 0.000000 & 1.078820 & 0.000000 \\ \mathrm{H} & 0.934286 & -0.539410 & 0.000000 \\ \mathrm{H} & -0.934286 & -0.539410 & 0.000000\end{array}$

Energy: -39.7609764

1.3.1 MPW1K/6-31+G(d,p) results:

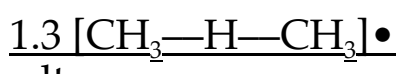

Cartesian Coordinates

$\begin{array}{lrrr}\mathrm{H} & 0.000000 & 0.000000 & 0.000000 \\ \mathrm{C} & 0.000000 & 0.000000 & 1.333452 \\ \mathrm{C} & 0.000000 & 0.000000 & -1.333452 \\ \mathrm{H} & 0.000000 & 1.045691 & 1.616159 \\ \mathrm{H} & 0.905585 & -0.522801 & 1.616159 \\ \mathrm{H} & -0.905585 & -0.522801 & 1.616159 \\ \mathrm{H} & 0.000000 & -1.045691 & -1.616159 \\ \mathrm{H} & -0.905585 & 0.522801 & -1.616159\end{array}$




\begin{tabular}{|c|c|c|c|}
\hline $\mathrm{H}$ & 0.905585 & 0.522801 & -1.616159 \\
\hline & $\begin{array}{l}:-80.321 \\
:-80.239\end{array}$ & & \\
\hline \multicolumn{4}{|c|}{ 1.3.2 CBS-QB3 results: } \\
\hline & & Cartesian & Coordinates \\
\hline $\mathrm{H}$ & 0.000000 & 0.000000 & 0.000000 \\
\hline $\mathrm{C}$ & 0.000000 & 0.000000 & 1.347086 \\
\hline $\mathrm{C}$ & 0.000000 & 0.000000 & -1.347086 \\
\hline $\mathrm{H}$ & 0.000000 & 1.049684 & 1.632260 \\
\hline $\mathrm{H}$ & 0.909053 & -0.524842 & 1.632260 \\
\hline $\mathrm{H}$ & -0.909053 & -0.524842 & 1.632260 \\
\hline $\mathrm{H}$ & 0.000000 & -1.049684 & -1.632260 \\
\hline $\mathrm{H}$ & -0.909053 & 0.524842 & -1.632260 \\
\hline $\mathrm{H}$ & 0.909053 & 0.524842 & -1.632260 \\
\hline
\end{tabular}

Energy: -80.200549

Enthalpy: -80.121796

\subsubsection{UCCSD(T)/ cc-pVTZ results:}

$\begin{array}{rrrr} & & \text { Cartesian } & \text { Coordinates } \\ \mathrm{H} & 0.000000 & 0.000000 & 0.000000 \\ \mathrm{C} & 0.000000 & 0.000000 & 1.340294 \\ \mathrm{C} & 0.000000 & 0.000000 & -1.340294 \\ \mathrm{H} & 0.000000 & 1.049009 & 1.623351 \\ \mathrm{H} & 0.908468 & -0.524504 & 1.623351 \\ \mathrm{H} & -0.908468 & -0.524504 & 1.623351 \\ \mathrm{H} & 0.000000 & -1.049009 & -1.623351 \\ \mathrm{H} & -0.908468 & 0.524504 & -1.623351 \\ \mathrm{H} & 0.908468 & 0.524504 & -1.623351\end{array}$

Energy: -80.1702689

1.4.1 MPW1K/6-31+G(d,p) results:

$\underline{1.4 \mathrm{NH}_{3}}$

$\begin{array}{rrrr} & & \text { Cartesian } & \text { Coordinates } \\ \mathrm{N} & 0.000000 & 0.000000 & 0.105380 \\ \mathrm{H} & 0.000000 & 0.942594 & -0.245886 \\ \mathrm{H} & 0.816311 & -0.471297 & -0.245886 \\ \mathrm{H} & -0.816311 & -0.471297 & -0.245886\end{array}$

Energy: -56.545716

Enthalpy: -56.506344

1.4.2 CBS-QB3 results: 


$\begin{array}{rrrr}\mathrm{N} & 0.000000 & 0.000000 & 0.115796 \\ \mathrm{H} & 0.000000 & 0.939191 & -0.270191 \\ \mathrm{H} & -0.813364 & -0.469596 & -0.270191 \\ \mathrm{H} & 0.813364 & -0.469596 & -0.270191\end{array}$

Energy: -56.494148

Enthalpy: -56.456360

1.4.3 CCSD(T)/ cc-pVTZ results:

$\begin{array}{lrrr} & & \text { Cartesian } & \text { Coordinates } \\ \mathrm{N} & 0.000000 & 0.000000 & 0.119182 \\ \mathrm{H} & 0.000000 & 0.933033 & -0.278092 \\ \mathrm{H} & 0.808030 & -0.466516 & -0.278092 \\ \mathrm{H} & -0.808030 & -0.466516 & -0.278092\end{array}$

Energy: -56.4731973

$\underline{\text { 1.5.1 MPW1K/6-31+G(d,p) results: }} \underline{1.5 \mathrm{NH}_{2}} \underline{-}$

$\begin{array}{rrrr} & & \text { Cartesian } & \text { Coordinates } \\ \mathrm{N} & 0.000000 & 0.000000 & 0.139198 \\ \mathrm{H} & 0.000000 & 0.804167 & -0.487192 \\ \mathrm{H} & 0.000000 & -0.804167 & -0.487192\end{array}$

Energy: -55.865356

Enthalpy: -55.841794

1.5.2 CBS-QB3 results:

$\begin{array}{rrrr} & & & \text { Cartesian } \\ \mathrm{N} & 0.000000 & 0.000000 & 0.143925 \\ \mathrm{H} & 0.000000 & 0.801913 & -0.503737 \\ \mathrm{H} & 0.000000 & -0.801913 & -0.503737\end{array}$

Energy: -56.809874

Enthalpy: -55.787405

1.5.3 UCCSD(T)/ cc-pVTZ results:

$\begin{array}{lrrr} & & \text { Cartesian } & \text { Coordinates } \\ \mathrm{N} & 0.000000 & 0.000000 & 0.143264 \\ \mathrm{H} & 0.000000 & 0.799318 & -0.501424 \\ \mathrm{H} & 0.000000 & -0.799318 & -0.501424\end{array}$

Energy: -55.7936266

1.6.1 MPW1K/6-31+G(d,p) results:

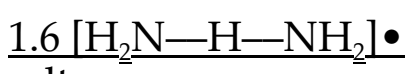

\begin{tabular}{rrrr}
\hline & & Cartesian Coordinates \\
$\mathrm{H}$ & 0.000000 & 0.000000 & 0.378463
\end{tabular}




$\begin{array}{rrrr}\mathrm{N} & 0.000000 & 1.203692 & 0.107396 \\ \mathrm{~N} & 0.000000 & -1.203692 & 0.107396 \\ \mathrm{H} & -0.810497 & 1.395138 & -0.470501 \\ \mathrm{H} & 0.810497 & 1.395138 & -0.470501 \\ \mathrm{H} & 0.810497 & -1.395138 & -0.470501 \\ \mathrm{H} & -0.810497 & -1.395138 & -0.470501\end{array}$

Energy: -112.393518

Enthalpy: -112.332670

1.6.2 CBS-QB3 results:

\begin{tabular}{rrrr}
\hline & & Cartesian & Coordinates \\
$\mathrm{H}$ & 0.000000 & 0.000000 & 0.380884 \\
$\mathrm{~N}$ & 0.000000 & 1.220269 & 0.112427 \\
$\mathrm{~N}$ & 0.000000 & -1.220269 & 0.112427 \\
$\mathrm{H}$ & -0.809777 & 1.391186 & -0.488715 \\
$\mathrm{H}$ & 0.809777 & 1.391186 & -0.488715 \\
$\mathrm{H}$ & 0.809777 & -1.391186 & -0.488715 \\
$\mathrm{H}$ & -0.809777 & -1.391186 & -0.488715
\end{tabular}

Energy: -112.287171

Enthalpy: -112.228826

\subsubsection{UCCSD(T)/ cc-pVTZ results:}

$\begin{array}{rrrr} & & \text { Cartesian } & \text { Coordinates } \\ \mathrm{H} & 0.000000 & 0.000000 & 0.388418 \\ \mathrm{~N} & 0.000000 & 1.213798 & 0.115826 \\ \mathrm{~N} & 0.000000 & -1.213798 & 0.115826 \\ \mathrm{H} & -0.805145 & 1.331762 & -0.502495 \\ \mathrm{H} & 0.805145 & 1.331762 & -0.502495 \\ \mathrm{H} & 0.805145 & -1.331762 & -0.502495 \\ \mathrm{H} & -0.805145 & -1.331762 & -0.502495\end{array}$

Energy: -112.2487192

1.7.1 MPW1K/6-31+G(d,p) results:

$\underline{1.7 \mathrm{H}_{2}} \underline{\mathrm{O}}$

\begin{tabular}{rrrr} 
& & Cartesian & \multicolumn{1}{r}{ Coordinates } \\
$\mathrm{O}$ & 0.000000 & 0.000000 & 0.114399 \\
$\mathrm{H}$ & 0.000000 & 0.762160 & -0.457596 \\
$\mathrm{H}$ & 0.000000 & -0.762160 & -0.457596
\end{tabular}

Energy: -76.403845

Enthalpy: -76.377855

1.7.2 CBS-QB3 results:

$\begin{array}{rrrr} & & \text { Cartesian Coordinates } \\ 0 & 0.000000 & 0.000000 & 0.118700\end{array}$




$\begin{array}{rrrr}\mathrm{H} & 0.000000 & 0.757091 & -0.474801 \\ \mathrm{H} & 0.000000 & -0.757091 & -0.474801\end{array}$

Energy: -76.358591

Enthalpy: -76.333712

1.7.3 CCSD(T)/cc-pVTZ results:

$\begin{array}{rrrr} & & \text { Cartesian } & \text { Coordinates } \\ \mathrm{O} & 0.000000 & 0.000000 & 0.118685 \\ \mathrm{H} & 0.000000 & 0.753881 & -0.474739 \\ \mathrm{H} & 0.000000 & -0.753881 & -0.474739\end{array}$

Energy: -76.3322165

1.8.1 MPW1K/6-31+G(d,p) results:

\section{$\underline{1.8 \mathrm{OH} \bullet}$}

Cartesian Coordinates

$\begin{array}{rrrr}\mathrm{O} & 0.000000 & 0.000000 & 0.107368 \\ \mathrm{H} & 0.000000 & 0.000000 & -0.858946\end{array}$

Energy: -75.712170

Enthalpy: -75.700002

$\underline{1.8 .2 ~ C B S-Q B 3 ~ r e s u l t s: ~}$

$\begin{array}{rrrr} & & \text { Cartesian } & \text { Coordinates } \\ \mathrm{O} & 0.000000 & 0.000000 & 0.108358 \\ \mathrm{H} & 0.000000 & 0.000000 & -0.866864\end{array}$

Energy: -75.658075

Enthalpy: -75.646416

$\underline{\text { 1.8.3 UCCSD(T)/ cc-pVTZ results: }}$

$\begin{array}{rrrr} & & \text { Cartesian } & \text { Coordinates } \\ \mathrm{O} & 0.000000 & 0.000000 & 0.107900 \\ \mathrm{H} & 0.000000 & 0.000000 & -0.863199\end{array}$

Energy: -75.6377235

1.9.1 MPW1K/6-31+G(d,p) results:

$$
1.9[\mathrm{HO}-\mathrm{H}-\mathrm{OH}] \bullet
$$

Cartesian Coordinates

$\begin{array}{rrrr}\mathrm{H} & 0.000000 & 0.000000 & 0.391739 \\ \mathrm{O} & 0.000000 & 1.093639 & 0.035283 \\ \mathrm{O} & 0.000000 & -1.093639 & 0.035283 \\ \mathrm{H} & -0.806007 & 1.190698 & -0.478131 \\ \mathrm{H} & 0.806007 & -1.190698 & -0.478131\end{array}$

Energy: -152.102293 
Enthalpy: -152.067444

1.9.2 CBS-QB3 results:

\begin{tabular}{rrrr}
\hline & & \multicolumn{1}{r}{ Cartesian } & Coordinates \\
$\mathrm{H}$ & 0.000000 & 0.000000 & 0.411957 \\
$\mathrm{O}$ & 0.000000 & 1.100875 & 0.029431 \\
$\mathrm{O}$ & 0.000000 & -1.100875 & 0.029431 \\
$\mathrm{H}$ & -0.842606 & 1.176852 & -0.441428 \\
$\mathrm{H}$ & 0.842606 & -1.176852 & -0.441428
\end{tabular}

Energy: -152.001674

Enthalpy: -151.967920

1.9.3 UCCSD(T)/cc-pVTZ results:

$\begin{array}{lrrr} & & \text { Cartesian } & \text { Coordinates } \\ \mathrm{H} & 0.000000 & 0.000000 & 0.419813 \\ \mathrm{O} & 0.000000 & 1.095453 & 0.034630 \\ \mathrm{O} & 0.000000 & -1.095453 & 0.034630 \\ \mathrm{H} & -0.814239 & 1.119739 & -0.486949 \\ \mathrm{H} & 0.814239 & -1.119739 & -0.486949\end{array}$

Energy: -151.9558151

1.10.1 MPW1K/6-31+G(d,p) results:

$\underline{1.10 \mathrm{HF}}$

Cartesian Coordinates

$\begin{array}{lrrr}\text { F } & 0.000000 & 0.000000 & 0.091452 \\ \mathrm{H} & 0.000000 & 0.000000 & -0.823068\end{array}$

Energy: -100.416474

Enthalpy: -100.403446

1.10.2 CBS-QB3 results:

$\begin{array}{lrrr} & & \text { Cartesian } & \text { Coordinates } \\ \mathrm{F} & 0.000000 & 0.000000 & 0.091986 \\ \mathrm{H} & 0.000000 & 0.000000 & -0.827870\end{array}$

Energy: $-100 \cdot 369214$

Enthalpy: -100.356619

1.10.3 CCSD(T)/cc-pVTZ results:

$\begin{array}{lrrr} & & \text { Cartesian } & \text { Coordinates } \\ \mathrm{F} & 0.000000 & 0.000000 & 0.091724 \\ \mathrm{H} & 0.000000 & 0.000000 & -0.825516\end{array}$

Energy: $-100 \cdot 3383564$

\section{$\underline{1.11 \mathrm{~F} \bullet}$}


1.11.1 MPW1K/6-31+G(d,p) results:

Energy: -99.702824

Enthalpy: -99.700464

1.11.2 CBS-QB3 results:

Energy: -99.643075

Enthalpy: -99.640715

1.11.3 UCCSD(T)/ cc-pVTZ results:

Energy: -99.6203608

1.12.1 MPW1K/6-31+G(d,p) results:

$$
1.12[\mathrm{~F}-\mathrm{H}-\mathrm{F}] \bullet
$$

Cartesian Coordinates

$\begin{array}{lrrr}\mathrm{H} & 0.000000 & 0.000000 & 0.400277 \\ \mathrm{~F} & 0.000000 & 1.010478 & -0.022238 \\ \mathrm{~F} & 0.000000 & -1.010478 & -0.022238\end{array}$

Energy: -200.093877

Enthalpy: -200.084928

1.12.2 CBS-QB3 results:

\begin{tabular}{lrrr} 
& & \multicolumn{1}{r}{ Cartesian } & Coordinates \\
$\mathrm{H}$ & 0.000000 & 0.000000 & 0.398624 \\
$\mathrm{~F}$ & 0.000000 & 1.023264 & -0.022146 \\
$\mathrm{~F}$ & 0.000000 & -1.023264 & -0.022146
\end{tabular}

Energy: -199.983786

Enthalpy: -199.975066

1.12.3 UCCSD(T)/cc-pVTZ results:

$\begin{array}{lrrr} & & \text { Cartesian } & \text { Coordinates } \\ \mathrm{H} & 0.000000 & 0.000000 & 0.418141 \\ \mathrm{~F} & 0.000000 & 1.008447 & -0.023230 \\ \mathrm{~F} & 0.000000 & -1.008447 & -0.023230\end{array}$

Energy: -199.9304217

1.13.1 MPW1K/6-31+G(d,p) results:

$$
\underline{1.13 \mathrm{H}_{2}} \underline{\mathrm{O}}_{2}
$$

Cartesian Coordinates

o $\quad 0.000000 \quad 0.705494 \quad-0.054588$

$\begin{array}{llll}0 & 0.000000 & -0.705494 & -0.054588\end{array}$

$\mathrm{H} \quad 0.799850 \quad 0.897021 \quad 0.436702$

$\begin{array}{llll}\mathrm{H} & -0.799850 & -0.897021 & 0.436702\end{array}$ 
Energy: -151.493186

Enthalpy: -151.461077

1.13.2 CBS-QB3 results:

$\begin{array}{rrrr} & & \text { Cartesian } & \text { Coordinates } \\ \mathrm{O} & 0.000000 & 0.726655 & -0.052496 \\ \mathrm{O} & 0.000000 & -0.726655 & -0.052496 \\ \mathrm{H} & 0.825379 & 0.896151 & 0.419969 \\ \mathrm{H} & -0.825379 & -0.896151 & 0.419969\end{array}$

Energy: -151.404994

Enthalpy: -151.374583

1.13.3 CCSD(T)/cc-pVTZ results:

\begin{tabular}{lrrr} 
& & \multicolumn{1}{c}{ Cartesian } & Coordinates \\
$\mathrm{O}$ & 0.000000 & 0.728926 & -0.057626 \\
$\mathrm{O}$ & 0.000000 & -0.728926 & -0.057626 \\
$\mathrm{H}$ & 0.796711 & 0.888900 & 0.461006 \\
$\mathrm{H}$ & -0.796711 & -0.888900 & 0.461006
\end{tabular}

Energy: -151.3586107

1.14.1 MPW1K/6-31+G(d,p) results:

\section{$\underline{1.14 \mathrm{HOO} \bullet}$}

Cartesian Coordinates

$\begin{array}{rrrr}\mathrm{O} & 0.054595 & -0.597276 & 0.000000 \\ \mathrm{O} & 0.054595 & 0.705282 & 0.000000 \\ \mathrm{H} & -0.873524 & -0.864044 & 0.000000\end{array}$

Energy: -150.850821

Enthalpy: -150.831991

1.14.2 CBS-QB3 results:

\begin{tabular}{rrrr} 
& & \multicolumn{1}{c}{ Cartesian } & Coordinates \\
$\mathrm{O}$ & 0.055270 & -0.609731 & 0.000000 \\
$\mathrm{O}$ & 0.055270 & 0.718659 & 0.000000 \\
$\mathrm{H}$ & -0.884314 & -0.871425 & 0.000000
\end{tabular}

Energy: -150.755045
Enthalpy: -150.737256

1.14.3 UCCSD(T)/cc-pVTZ results:

$\begin{array}{rrrr} & & \text { Cartesian } & \text { Coordinates } \\ \mathrm{O} & 0.055483 & -0.615166 & 0.000000 \\ \mathrm{O} & 0.055483 & 0.721226 & 0.000000 \\ \mathrm{H} & -0.887720 & -0.848479 & 0.000000\end{array}$


Energy: -150.7127218

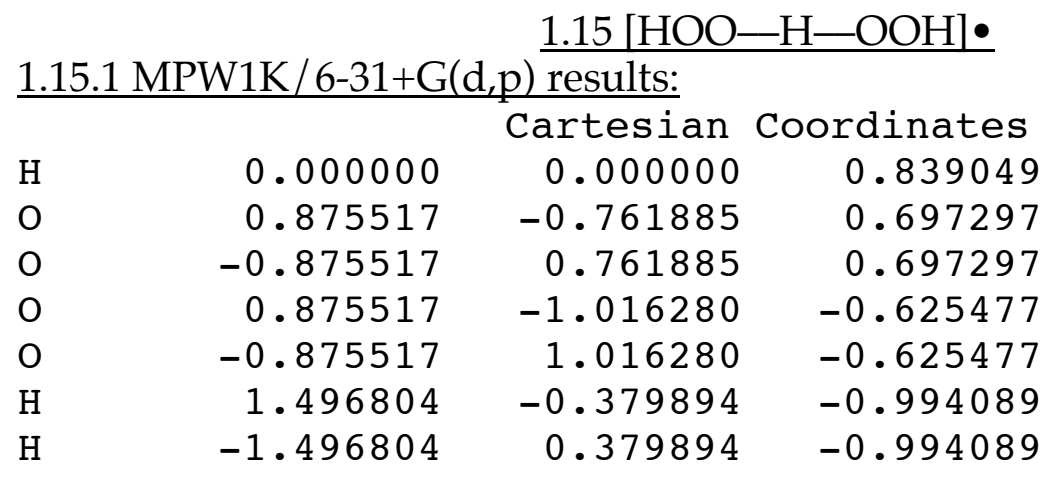

Energy: -302.324033

Enthalpy: -302.277308

1.15.2 CBS-QB3 results:

\begin{tabular}{rrrr}
\hline & & Cartesian & Coordinates \\
$\mathrm{H}$ & 0.000000 & 0.000000 & 0.853223 \\
$\mathrm{O}$ & 0.918158 & -0.731572 & 0.714323 \\
$\mathrm{O}$ & -0.918158 & 0.731572 & 0.714323 \\
$\mathrm{O}$ & 0.918158 & -0.990428 & -0.641797 \\
$\mathrm{O}$ & -0.918158 & 0.990428 & -0.641797 \\
$\mathrm{H}$ & 1.502444 & -0.305695 & -1.006817 \\
$\mathrm{H}$ & -1.502444 & 0.305695 & -1.006817
\end{tabular}

Energy: -302.142637

Enthalpy: -302.098373

1.15.3 UCCSD(T)/ cc-pVTZ results:

$\begin{array}{rrrr} & & \text { Cartesian } & \text { Coordinates } \\ \mathrm{H} & 0.000000 & 0.000000 & 0.862621 \\ \mathrm{O} & 1.024094 & -0.565638 & 0.717887 \\ \mathrm{O} & -1.024094 & 0.565638 & 0.717887 \\ \mathrm{O} & 1.024094 & -0.821485 & -0.645699 \\ \mathrm{O} & -1.024094 & 0.821485 & -0.645699 \\ \mathrm{H} & 1.430651 & -0.019455 & -1.008816 \\ \mathrm{H} & -1.430651 & 0.019455 & -1.008816\end{array}$

Energy: -302.0507247

1.16.1 MPW1K/6-31+G(d,p) results:

\section{$1.16 \mathrm{H}_{2} \underline{\mathrm{NOH}}$}

Cartesian Coordinates

$\begin{array}{lrrr}\mathrm{O} & -0.008786 & -0.720582 & 0.000000 \\ \mathrm{~N} & -0.008786 & 0.687703 & 0.000000 \\ \mathrm{H} & -0.935866 & -0.946725 & 0.000000 \\ \mathrm{H} & 0.533829 & 0.948728 & 0.810501\end{array}$




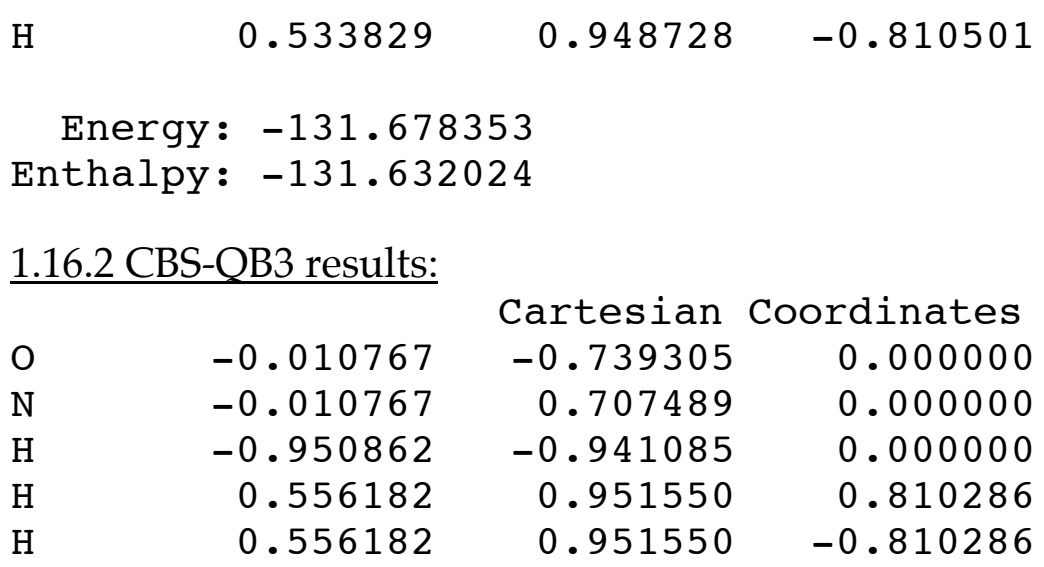

Energy: -131.581484

Enthalpy: -131.537437

1.16.3 CCSD $(\mathrm{T}) / \mathrm{cc}-\mathrm{pVTZ}$ results:

\begin{tabular}{lrrr} 
& & \multicolumn{1}{c}{ Cartesian } & Coordinates \\
$\mathrm{O}$ & -0.011507 & -0.739637 & 0.000000 \\
$\mathrm{~N}$ & -0.011507 & 0.708976 & 0.000000 \\
$\mathrm{H}$ & -0.952189 & -0.929817 & 0.000000 \\
$\mathrm{H}$ & 0.562398 & 0.942043 & 0.806100 \\
$\mathrm{H}$ & 0.562398 & 0.942043 & -0.806100
\end{tabular}

Energy: -131.5381826

1.17.1 MPW1K/6-31+G(d,p) results:

\section{$\underline{1.17 \mathrm{H}_{2}} \underline{\mathrm{NO} \bullet}$}

Cartesian Coordinates

$\begin{array}{rrrr}\mathrm{O} & -0.009040 & -0.726701 & 0.000000 \\ \mathrm{~N} & -0.009040 & 0.535508 & 0.000000 \\ \mathrm{H} & 0.067796 & 1.032529 & 0.872048 \\ \mathrm{H} & 0.067796 & 1.032529 & -0.872048\end{array}$

Energy: -131.050071

Enthalpy: -131.018258

1.17.2 CBS-QB3 results:

$\begin{array}{rrrr} & & \text { Cartesian } & \text { Coordinates } \\ \mathrm{O} & 0.017057 & -0.734757 & 0.000000 \\ \mathrm{~N} & 0.017057 & 0.541917 & 0.000000 \\ \mathrm{H} & -0.127930 & 1.042316 & 0.873595 \\ \mathrm{H} & -0.127930 & 1.042316 & -0.873595\end{array}$

Energy: -130.946741

Enthalpy: -130.916378

1.17.3 UCCSD(T)/ cc-pVTZ results: 


$\begin{array}{lrrr} & & \text { Cartesian } & \text { Coordinates } \\ \mathrm{O} & 0.022022 & -0.734228 & 0.000000 \\ \mathrm{~N} & 0.022022 & 0.545035 & 0.000000 \\ \mathrm{H} & -0.165164 & 1.029290 & 0.869200 \\ \mathrm{H} & -0.165164 & 1.029290 & -0.869200\end{array}$

Energy: -130.9041855

1.18.1 MPW1K/6-31+G(d,p) results:

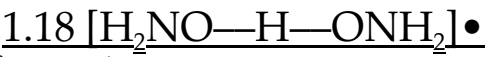

Cartesian Coordinates

$\begin{array}{lrrr}\mathrm{H} & 0.000000 & 0.000000 & 0.858330 \\ \mathrm{O} & 0.000000 & 1.165390 & 0.709317 \\ \mathrm{O} & 0.000000 & -1.165390 & 0.709317 \\ \mathrm{~N} & 0.000000 & 1.329316 & -0.600940 \\ \mathrm{~N} & 0.000000 & -1.329316 & -0.600940 \\ \mathrm{H} & -0.845601 & 1.752446 & -0.948560 \\ \mathrm{H} & 0.845601 & -1.752446 & -0.948560 \\ \mathrm{H} & 0.845601 & 1.752446 & -0.948560 \\ \mathrm{H} & -0.845601 & -1.752446 & -0.948560\end{array}$

Energy: -262.707855

Enthalpy: -262.632932

$\underline{1.18 .2 \text { CBS-QB3 results: }}$

$\begin{array}{lrrr} & & \text { Cartesian } & \text { Coordinates } \\ \mathrm{H} & 0.000000 & 0.000000 & 0.831895 \\ \mathrm{O} & 0.000000 & 1.178230 & 0.715673 \\ \mathrm{O} & 0.000000 & -1.178230 & 0.715673 \\ \mathrm{~N} & 0.000000 & 1.394904 & -0.611675 \\ \mathrm{~N} & 0.000000 & -1.394904 & -0.611675 \\ \mathrm{H} & -0.847232 & 1.857549 & -0.929804 \\ \mathrm{H} & 0.847232 & -1.857549 & -0.929804 \\ \mathrm{H} & 0.847232 & 1.857549 & -0.929804 \\ \mathrm{H} & -0.847232 & -1.857549 & -0.929804\end{array}$

Energy: -262.511962

Enthalpy: -262.440912

1.18.3 UCCSD(T)/ cc-pVTZ results:

\begin{tabular}{rrrr}
\hline & & Cartesian & Coordinates \\
$\mathrm{H}$ & 0.000000 & 0.000000 & 0.866564 \\
$\mathrm{O}$ & 0.000000 & 1.174712 & 0.724694 \\
$\mathrm{O}$ & 0.000000 & -1.174712 & 0.724694 \\
$\mathrm{~N}$ & 0.000000 & 1.301987 & -0.616782 \\
$\mathrm{~N}$ & 0.000000 & -1.301987 & -0.616782 \\
$\mathrm{H}$ & -0.844333 & 1.747658 & -0.956679 \\
$\mathrm{H}$ & 0.844333 & -1.747658 & -0.956679
\end{tabular}




$\begin{array}{rrrr}\mathrm{H} & 0.844333 & 1.747658 & -0.956679 \\ \mathrm{H} & -0.844333 & -1.747658 & -0.956679\end{array}$

Energy: -262.4192959

2. Comparison with Experimental Data

All of the experimental data are given in the form of Arrhenius activation energies, $E_{a}$. Since the reaction is a bimolecular, gas-phase process, the activation enthalpy, $\Delta H^{\ddagger}$, should be $2 R T$ lower than $E_{a}$, where $T$ is the mean temperature of the experimental range. This calculation was the source of the $\Delta H^{\ddagger}$ values in the table below. All values are in $\mathrm{kcal} / \mathrm{mol}$. One sees that for both the methyl and hydroxyl self exchanges, the experimental $\Delta H^{\ddagger}$ are lower than the calculated ones by several $\mathrm{kcal} / \mathrm{mol}$. Possibly this is an indication that tunneling plays an important role in the reaction. That issue is discussed explicitly in references 10, 11 and 12.

$$
\underline{2.1 \mathrm{H}_{3}} \underline{\mathrm{C} \cdot+\mathrm{H}-\mathrm{CH}_{3}} \rightarrow \mathrm{H}_{3} \underline{\mathrm{C}-\mathrm{H}+\bullet \mathrm{CH}_{3}}
$$

\begin{tabular}{ccccc} 
Exptl. $\mathrm{E}_{\mathrm{a}}$ & Exptl. $\boldsymbol{\Delta} \boldsymbol{H}^{\ddagger}$ & MPW1K $\boldsymbol{\Delta} \boldsymbol{H}^{\ddagger}$ & $\mathrm{CBS}-\mathrm{QB} 3 \boldsymbol{\Delta} \boldsymbol{H}^{\ddagger}$ & Reference \\
\hline 14.0 & 12.4 & 15.2 & 15.8 & 6 \\
14.65 & 13.5 & & & 7 \\
14.9 & 13.8 & & & 8 \\
14.0 & 11.5 & & & 9
\end{tabular}

A very extensive study of methods for calculating barriers to HAT reactions is reported in reference 10 . In that work, the authors deduce a classical barrier height of $17.5 \mathrm{kcal} / \mathrm{mol}$ for the $\mathrm{H}_{3} \mathrm{C} \bullet+\mathrm{H}-\mathrm{CH}_{3}$ reaction (excluding $\mathrm{ZPE}$ ).

$$
2.2 \mathrm{HO} \bullet+\mathrm{H}-\mathrm{OH} \rightarrow \mathrm{HO}-\mathrm{H}+\bullet \mathrm{OH}
$$

Only a single estimate of the activation barrier to this reaction has been reported. ${ }^{11}$ It leads to an Arrhenius activation energy of $4.2 \mathrm{kcal} / \mathrm{mol}$, corresponding to a $\Delta H^{\ddagger}$ value of $2.8 \mathrm{kcal} / \mathrm{mol}$. The MPW1K and CBS-QB3 $\Delta H^{\ddagger}$ values are 6.5 and $7.7 \mathrm{kcal} / \mathrm{mol}$, respectively. It seems unlikely that this discrepancy could be due to errors in the two very different computational models. Although no $\Delta H^{\ddagger}$ could be calculated at the UCCSD(T)/cc-pVTZ level, the PE barrier found by this method was very similar to those from the MPW1K and CBS-QB3 models. A careful computational study by Uchimaru et al. ${ }^{12}$ found a similar discrepancy, but showed that it could be resolved inclusion of a tunneling correction.

3. Parameters Used in the Existing Theoretical Models

The model of Zavitsas ${ }^{13}$ uses 8 input parameters, 4 each for the $\mathrm{X}-\mathrm{H}$ and $\mathrm{X}-\mathrm{X}$ bonds. They are the bond-dissociation enthalpies $\left(\mathrm{BDE}_{\mathrm{X}-\mathrm{H}}\right.$ and $\mathrm{BDE}_{\mathrm{X}-\mathrm{X}}$, in $\mathrm{kcal} / \mathrm{mol})$, stretching frequencies $\left(v_{X_{-H}}\right.$ and $v_{X-X}$, in $\left.\mathrm{cm}^{-1}\right)$, reduced masses $\left(\mu_{X-H}\right.$ and $\mu_{x_{-} X}$ in amu), and equilibrium bondlengths $\left(r_{x_{-H}}\right.$ and $r_{X_{-} x}$ in $\AA$ ). The values used for the six reactions considered in the paper are tabulated below. Most come from the NIST website (http:// webbook.nist.gov/chemistry/), but some 
come from other sources. Specifically, the N-N stretching frequency in hydrazine comes from reference 14, the $\mathrm{O}-\mathrm{O}$ bondlength and dissociation enthalpy in $\mathrm{HOO}-\mathrm{OOH}$ come from references 15 and 16 , and the $\mathrm{O}-\mathrm{O}$ bondlength and dissociation enthalpy in $\mathrm{H}_{2} \mathrm{NO}-\mathrm{ONH}_{2}$ come from a MPW1K/ 6$31+G(d)$ calculation done by the present authors.

\begin{tabular}{ccccrcccc}
$\mathrm{X}$ & $\mathrm{BDE}_{\mathrm{X}-\mathrm{H}}$ & $\mathrm{BDE}_{\mathrm{X}-\mathrm{X}}$ & $v_{\mathrm{X}-\mathrm{H}}$ & $v_{\mathrm{X}-\mathrm{X}}$ & $\mu_{\mathrm{X}-\mathrm{H}}$ & $\mu_{\mathrm{X}-\mathrm{X}}$ & $\mathrm{r}_{\mathrm{X}-\mathrm{H}}$ & $\mathrm{r}_{\mathrm{X}-\mathrm{X}}$ \\
\hline $\mathrm{CH}_{3}$ & 105.0 & 88.9 & 2994 & 995 & 0.9297 & 6.0000 & 1.087 & 1.535 \\
$\mathrm{NH}_{2}$ & 107.6 & 66.2 & 3388 & 1077 & 0.9402 & 7.0015 & 1.012 & 1.449 \\
$\mathrm{OH}$ & 118.8 & 50.3 & 3707 & 878 & 0.9481 & 7.9975 & 0.9575 & 1.464 \\
$\mathrm{~F}$ & 136.3 & 37.7 & 4139 & 892 & 0.9571 & 9.4992 & 0.9169 & 1.412 \\
$\mathrm{OOH}$ & 87.8 & 17.8 & 3614 & 955 & 0.9481 & 7.9975 & 0.9588 & 1.374 \\
$\mathrm{ONH}_{2}$ & 77.4 & 19.3 & 3840 & 656 & 0.9481 & 7.9975 & 0.9597 & 1.428
\end{tabular}

The model of Roberts and Stee ${ }^{17}$ uses four constants, whose values are determined from least-squares best fit to existing experimental data. They are $E_{0}$ $=38.337 \mathrm{~kJ} \mathrm{~mol}^{-1}, \alpha=0.2472, \beta=-2.048 \mathrm{~kJ} \mathrm{~mol}^{-1} \mathrm{eV}^{-2}$, and $\gamma=3.412 \mathrm{~kJ} \mathrm{~mol}^{-1}$. The predicted activation energy for a HAT reaction between $\mathrm{AH}$ and $\bullet \mathrm{B}$ is then given by:

$$
\begin{aligned}
E_{a} & =E_{0} f+\alpha \Delta H^{\circ}(1-d)+\beta \Delta \chi_{A B}^{2}+\gamma\left(s_{A}+s_{B}\right) \\
f & =\left(D_{A H} D_{B H} / D_{H_{2}}^{2}\right)
\end{aligned}
$$

where $D_{A H}, D_{B H}$, and $D_{H 2}$ are the bond dissociation enthalpies for $\mathrm{AH}, \mathrm{BH}$, and $\mathrm{H}_{2}$, respectively, $\Delta H^{\circ}$ is the enthalpy of reaction, $d$ is a delocalization term for radical $\mathrm{B} \bullet, \Delta \chi_{A B}$ is the difference in electronegativities of $\mathrm{A} \bullet$ and $\mathrm{B} \bullet$, and $s_{A}$ and $s_{B}$ are structural parameters characteristic of $\mathrm{A} \bullet$ and $\mathrm{B} \bullet$. For the special case of degenerate HAT reactions, the second and third terms of the equation for $E_{a}$ are zero, and $D_{A H}=D_{B H}=\mathrm{BDE}_{\mathrm{X}-\mathrm{H}}$ from the table above. With $D_{H 2}=104.2 \mathrm{kcal} / \mathrm{mol}$, the quantity $f$ thus takes on the values 1.0154, 1.0663, 1.2999, 1.7110, 0.7100, and 0.5518 for $\mathrm{X}=\mathrm{CH}_{3}, \mathrm{NH}_{2}, \mathrm{OH}, \mathrm{F}, \mathrm{OOH}$, and $\mathrm{ONH}_{2}$ respectively. The correct value for $s_{A}\left(=s_{B}\right)$ is uncertain in the cases of $\mathrm{OOH}$ and $\mathrm{ONH}_{2}$, and so the range of values for $E_{a}$ in Table 2 of the main text reflects the range of possible values for $s$ $(0-0.6)$ given by Roberts and Stee ${ }^{17}$ for heteroatom-centered radicals.

The model of Shaik et al. (equation 22 in reference 18) is the simplest of all. It proposes that the barrier to degenerate HAT reactions is a constant fraction of the $\mathrm{X}-\mathrm{H} \mathrm{BDE}$, where the proportionality constant is 0.1667 .

4. Other Electronic-Structure Calculations on the Reactions Considered Here There have been scattered calculations on some of the $\mathrm{X}-\mathrm{H}-\mathrm{X}$ transition states considered in the present paper. The only other systematic comparison of which we are aware is the study by Leroy et al. ${ }^{19}$ However, the calculations leading to were conducted only at the UHF/6-31G level (with larger basis-set CI singlepoint corrections to the energies). They led to geometries and barrier heights substantially out of line with later higher-level calculations. Basch and $\mathrm{Hoz}^{20}$ have looked at the series $\mathrm{X}=\mathrm{CH}_{3}, \mathrm{NH}_{2}$, and $\mathrm{OH}$ at levels up to 
CCSD(T)/ /UMP2/6-311++G(2d,p). Since they excluded the F + H-F reaction, they did not detect the apparent anomaly in its barrier.

Davidson has recently carried out high-level calculations on $\mathrm{F}-\mathrm{H}-\mathrm{F}$, but only the linear structure was considered. ${ }^{21}$ The apparently anomalous barrier to the $\mathrm{F}+$ $\mathrm{H}-\mathrm{F}$ reaction was first pointed out over 30 years ago by $\mathrm{O}^{\prime} \mathrm{Neil}$ et al. ${ }^{22}$, but, again, that was a computational prediction based on a linear $\mathrm{F}-\mathrm{H}-\mathrm{F}$ transition structure. Fox and Schlegel ${ }^{23}$ carried out calculations on the F-H-F TS at levels up to QCISD(T)/D95++(3df,2p). At this level they found a geometry and barrier height very similar to ours. They also pointed out that lower-level methods can erroneously find a $C_{2 v}$ local minimum because of inadequate treatment of avoided crossings that occur near the TS.

The $\mathrm{HO}+\mathrm{H}-\mathrm{OH}$ reaction has been studied computationally by Masgrau et al. ${ }^{24}$ at levels up to CCSD/6-311G(3d,2p). They found a transition structure and barrier height very similar to ours, and again concluded that tunneling must be very important.

The $\mathrm{H}_{2} \mathrm{~N}+\mathrm{H}-\mathrm{NH}_{2}$ reaction has been studied computationally by Mebel et al. ${ }^{25}$ using a variety of modified G2 methods. They found transition structures very similar to ours, and classical barriers of $12.5-13.2 \mathrm{kcal} / \mathrm{mol}$.

A variety of calculations have been carried out on the $\mathrm{H}_{3} \mathrm{C}+\mathrm{H}-\mathrm{CH}_{3}$ reaction. All come to conclusions about the transition structure and barrier that are similar to ours. ${ }^{10,26-28}$

Calculations on self-exchange HAT reactions between heavier halogens are extensive. The first to show that the $\mathrm{Cl}-\mathrm{H}-\mathrm{Cl}$ is bent is in ref. 29 , while an early comparison of anionic and neutral transition structures can be found in ref. 30 .

\section{References}

1. Gaussian 03, Revision B.05, Frisch, M. J.; Trucks, G. W.; Schlegel, H. B.; Scuseria, G. E.; Robb, M. A.; Cheeseman, J. R.; Montgomery, Jr., J. A.; Vreven, T.; Kudin, K. N.; Burant, J. C.; Millam, J. M.; Iyengar, S. S.; Tomasi, J.; Barone, V.; Mennucci, B.; Cossi, M.; Scalmani, G.; Rega, N.; Petersson, G. A.; Nakatsuji, H.; Hada, M.; Ehara, M.; Toyota, K.; Fukuda, R.; Hasegawa, J.; Ishida, M.; Nakajima, T.; Honda, Y.; Kitao, O.; Nakai, H.; Klene, M.; Li, X.; Knox, J. E.; Hratchian, H. P.; Cross, J. B.; Bakken, V.; Adamo, C.; Jaramillo, J.; Gomperts, R.; Stratmann, R. E.; Yazyev, O.; Austin, A. J.; Cammi, R.; Pomelli, C.; Ochterski, J. W.; Ayala, P. Y.; Morokuma, K.; Voth, G. A.; Salvador, P.; Dannenberg, J. J.; Zakrzewski, V. G.; Dapprich, S.; Daniels, A. D.; Strain, M. C.; Farkas, O.; Malick, D. K.; Rabuck, A. D.; Raghavachari, K.; Foresman, J. B.; Ortiz, J. V.; Cui, Q.; Baboul, A. G.; Clifford, S.; Cioslowski, J.; Stefanov, B. B.; Liu, G.; Liashenko, A.; Piskorz, P.; Komaromi, I.; Martin, R. L.; Fox, D. J.; Keith, T.; Al-Laham, M. A.; Peng, C. Y.; Nanayakkara, A.; Challacombe, M.; Gill, P. M. W.; Johnson, B.; Chen, W.; Wong, M. W.; Gonzalez, C.; and Pople, J. A.; Gaussian, Inc., Wallingford CT, 2004. 
2. Lynch, B. J.; Fast, P. L.; Harris, M.; Truhlar, D. G. J. Phys. Chem. A, 2000, 104, $4811-5$.

3. (a) Ditchfield, R.; Hehre, W. J.; Pople, J. A. J. Chem. Phys. 1971, 54, 724-8. (b) Clark, T.; Chandrasekhar, J.; Spitznagel, G. W.; Schleyer, P. v. R. J. Comput. Chem. 1983, 4, 294-301.

4. Ochterski, J. W.; Petersson, G. A. J. Chem. Phys. 1996, 104, 2598-619.

5. Dunning, T. H. J. Chem. Phys. 1989, 90, 1007-23.

6. McNesby, J. R.; Gordon, A. S. J. Am. Chem. Soc. 1954, 76, 4196-8.

7. Dainton, F. S.; Ivin, K. J.; Wilkinson, F. Trans. Faraday Soc. 1959, 55, 929-36.

8. Creak, G. A.; Dainton, F. S.; Ivin, K. J. Trans. Faraday Soc. 1962, 58, 326-32.

9. Kerr, J. A.; Parsonage, M. J. Evaluated Kinetic Data on Gas Phase Hydrogen Transfer reactions of Methyl Radicals, Butterworths, London, 1976.

10. Dybala-Defratyka, A.; Paneth, P.; Pu, J.; Truhlar, D. G. J. Phys. Chem. A 2004, 108, 2475-86.

11. Dubey, M. K.; Mohrschladt, R.; Donahue, N. M.; Anderson, J. G. J. Phys. Chem. A 1997, 101, 1494-500.

12. Uchimaru, T.; Chandra, A. K.; Tsuzuki, S.; Sugie, M.; Sekiya, A. J. Comput. Chem. 2003, 24, 1538-48.

13. Zavitsas, A. A.; Chatgilialoglu, C. J. Am. Chem. Soc. 1995, 117, 10645-54.

14. Gulaczyk, I.; Krezglewski, K.; Valentin, A. J. Mol. Spec. 2003, 220, 132-6.

15. Fermann, J. T.; Hoffman, B. C.; Tschumper, G. S.; Schaefer, H. F., III J. Chem. Phys. 1997, 106, 5102-8.

16. Mckay, D. J.; Wright, J. S. J. Am. Chem. Soc. 1998, 120, 1003-13.

17. Roberts, B. P.; Steel, A. J. J. Chem. Soc., Perkin Trans 2 1994, 2155-62.

18. Shaik, S.; Wu, W.; Dong, K.; Song, L.; Hiberty, P. C. J. Phys. Chem. A 2001, 105, 8226-35.

19. Leroy, G.; Sana, M.; Tinant, A. Can. J. Chem. 1985, 63, 1447-56.

20. Basch, H.; Hoz, S. J. Phys. Chem. A 1997, 101, 4416-31.

21. Davidson, E. R. Int. J. Quantum Chem. 2004, 98, 317-24.

22. O'Neil, S. V.; Schaefer, H. F., III; Bender, C. F. Proc. Natl. Acad. Sci. (USA) 1974, 71, 104-6.

23. Masgrau, L.; Gonzalez-Lafont, A.; Lluch, J. M. J. Phys. Chem. A 1999, 103, 1044-53.

24. Fox, G. L; Schlegel, H. B. J. Am. Chem. Soc. 1993, 115, 6870-6.

25. Mebel, A. M.; Moskaleva, L. V.; Lin, M. C. J. Mol. Struct. (THEOCHEM.) 1999, 461, 223-38.

26. Saeys, M.; Reyniers, M. F.; Marin, G. B.; Van Speybroeck, V.; Waroquier, M.; J. Phys. Chem. A 2003, 107, 9147-59.

27. Coote, M. L.; Collins, M. A.; Radom, L.; Mol. Phys. 2003, 101, 1329-38.

28. Wildman, T. A. Chem. Phys. Lett. 1986, 126, 325-9.

29. Garrett, B. C.; Truhlar, D. G.; Wagner, A. F.; Dunning, T. H., Jr., J. Chem. Phys. 1983, 78, 4400-13.

30. Truhlar, D. G.; Olson, P. C.; Parr C. A., J. Chem. Phys. 1972, 57, 4479-83. 